\title{
Right prefrontal cortex is activated for perceiving postural limits: a functional near-infrared spectroscopy study
}

\author{
Noriyuki Kamata ${ }^{1}$, Yoshimi Matsuo ${ }^{2}$, Ayako Matsuya ${ }^{3}$, Satoru Inoue ${ }^{1}$, Kazuo Abe ${ }^{3,4}$ \\ ${ }^{1}$ Rehabilitation Unit, Osaka University Hospital, Osaka, Japan; nkamata@hp-rehab.med.osaka-u.ac.jp \\ ${ }^{2}$ Faculty of Rehabilitation, Kobe Gakuin University, Hyogo, Japan \\ ${ }^{3}$ Faculty of Nursing and Rehabilitation, Konan Women's University, Hyogo, Japan \\ ${ }^{4}$ Department of Neurology and Rehabilitation Center, Konan Hospital, Hyogo, Japan
}

Received 25 September 2009; revised 26 October 2009; accepted 27 October 2009.

\begin{abstract}
The purpose of this study was to investigate neuronal mechanisms active during the perception of forward postural limits in a standing position and to specify fall-related brain activity using optical functional near-infrared spectroscopy. The study group included six right-handed, healthy female volunteers (range: 19, 20 years). The optical imaging device comprised 16 optodes designed to provide 24-channel recording of changes in hemoglobin oxygenation. We measured the changes of oxygenated hemoglobin levels in the frontal region when subjects perceived reachability in a standing position. Compared with those in other regions, the oxygenated hemoglobin levels in the right frontal region compatible with the right prefrontal cortex significantly increased. This result suggests that brain activities in the right prefrontal cortex are related to perception of reachability. Overestimation of postural limits has been reported as one of the risk factor for falling. This overestimation might be induced by dysfunction in the prefrontal cortex, resulting in a failure to inhibit a motor program that would have caused a loss of balance in reaching. Activation of the right prefrontal cortex may be a key factor for preventing accidental falls in the elderly and in patients with neurological disorders.
\end{abstract}

Keywords: Postural Limits; Falls; Functional Near-Infrared Spectroscopy (fNIRS); Prefrontal Cortex (PFC)

\section{INTRODUCTION}

Most people can appropriately perceive reachability, which is defined as the distance to which one could reach if actually executing the reach. Previous studies often utilized forward perceived reachability in a standing position as an index of one's own perceived postural limits [1-3]. Reaching movements are programmed based on this perception, and one can usually execute these movements safely by keeping the center of mass within postural limits [4].

Our previous study and others have reported that elderly and neurological patients, including patients with Parkinson's disease, tended to overestimate their reachability when standing, and thus, overestimate their own postural limits, even if a target is not within actual reach $[3,5]$. In addition, this overestimation has been reported as one of the risk factors of multiple falls [6]. Therefore, improving the perception of reachability might decrease the number of accidental falls in the elderly and in neurological patients. For creating a rationale for this new intervention, it is useful to investigate the neuronal mechanisms of this abnormal estimation of reachability. However, no research has yet examined such brain activities with regard to perception of reachability.

Functional near-infrared spectroscopy (fNIRS) can evaluate cortical activity by measuring the changes of hemoglobin oxygenation of blood within a few centimeters of the skull surface [7]. We adapted this technique to our study since it was suitable for use during dynamic tasks such as walking and in a standing position $[8,9]$. Thus, the purpose of this study was to clarify neuronal mechanism during the perception of forward postural limits in a standing position by fNIRS and to specify fall-related brain regions.

\section{METHODS}

\subsection{Subjects}

The study group included six right-handed, healthy females (range: 19, 20 years). Informed consent was obtained form each subject in accordance with the Helsinki Declaration. 


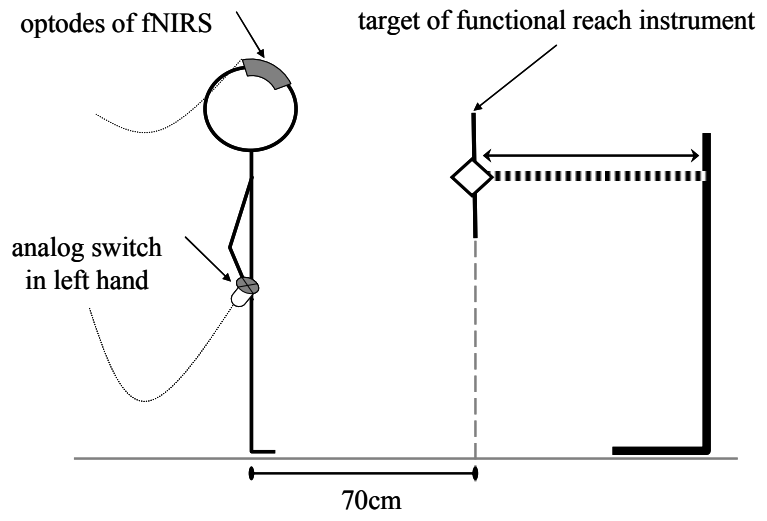

Figure 1. Position of subject and experimental devices. The target of the functional reach instrument can be moved along the dotted line. The distance from the target to the subject is $70 \mathrm{~cm}$.
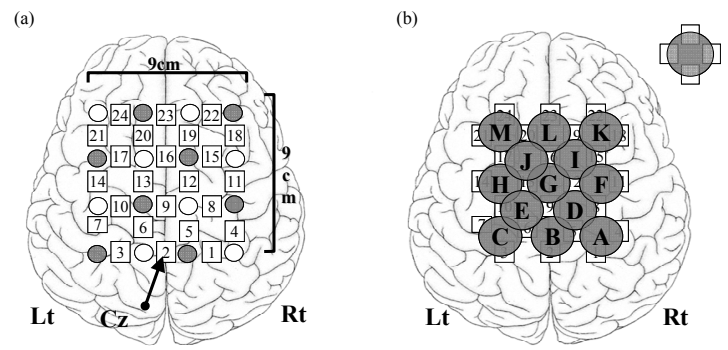

Figure 2. (a) Schema for location of the array of optodes. The optode array consists of 8 light fibers (white circles) and 8 detectors (black circles). The change of dortical hemoglobin content was measured in each channel between a light fiber and a detector (square numbers from 1 to 24 ). The center of the 4th row of optodes (channel 2) was located in the $\mathrm{Cz}$ position of the international 10-20 system. (b) Location of 13 regions of interest (circles labeled A-M). Each region consists of 4 nearest-neighbor channels For example, region of interest A was formed by channels $1,4,5$, and 8 .

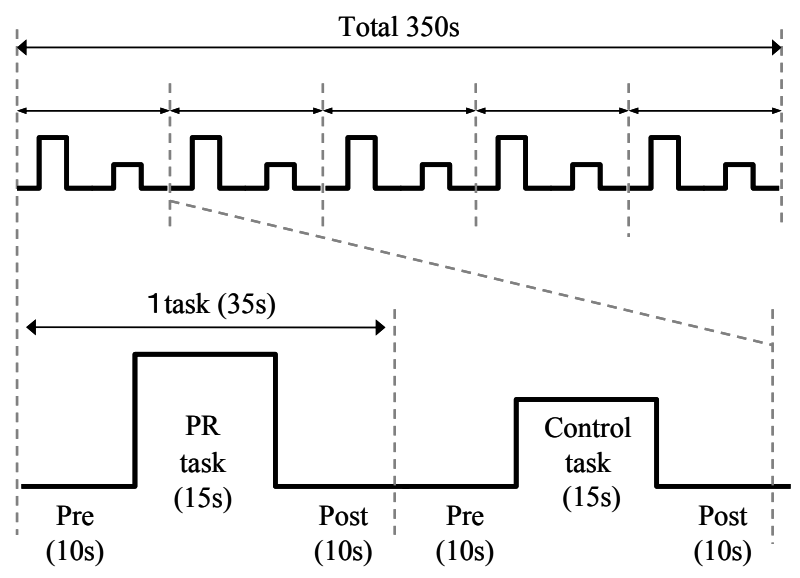

Figure 3. Task design for fNIRS. The perceived reachability tasks (taller blocks) and control tasks (lower blocks) were measured alternately and repeated continuously 5 times.

\subsection{Experimental Devices}

We used an optical imaging device (FOIRE3000, Shimadzu, Kyoto, Japan) and a standard device for the functional reach test [10] (GB-200, OG Giken, Okayama, Japan) with a wired analog switch. Subjects stood before the target of the functional reach test instrument with their feet positioned shoulder-width apart, holding the analog switch in their left hand (Figure 1).

\section{3. fNIRS}

The schema for location of the array of optodes in optical imaging device is showed in Figure 2a. The device consisted of 16 optodes, including 8 light-source fibers and 8 detectors arranged in a $4 \times 4$ array with the interoptode distance set at $3.0 \mathrm{~cm}$. We located the center of the 4th row of optodes in the $\mathrm{Cz}$ position, in accordance with to the international electroencephalogram 10-20 system. This configuration permitted 24-channel recording of changes in oxygenated hemoglobin (oxy-Hb), deoxygenated hemoglobin (deoxy-Hb), and total hemoglobin (total- $\mathrm{Hb})$ in the motor-related cortex within the covered $9 \times 9-\mathrm{cm}$ skull surface.

\subsection{Task Design and Experimental Protocol}

The task design for this fNIRS study is shown in Figure 3. We conducted two tasks, the perceived reachability task and the control task. Each task took a total of 35 seconds and consisted of a 10 -second pretask period, a 15 -second task period, and a 15 -second posttask period. The two tasks were measured alternately, and the protocol was repeated 5 times. Total duration for each fNIRS measurement session was 350 seconds.

During the tasks, the subject was instructed to concentrate on the target while in a standing position with arms at her sides and maintaining a static posture. In the perceived reachability task, the examiner moved the target in a direction away from the subject during the 15 -second test. When the subject judged that the target had arrived at the reachable limit for her right arm, she pushed the analog switch to record her perception. In control tasks, the examiner moved the target in a direction nearer to the subject.

\subsection{Data Analysis}

The hemoglobin oxygenation data was sampled every $160 \mathrm{~ms}$. We used the change of oxy-Hb level $(\mathrm{mM}-\mathrm{cm})$ as the index of cortical activity. When cortical activity increased, cortex needed more oxygen that increases oxy$\mathrm{Hb}$ level and it correlated better with the change of regional cerebral blood flow than deoxy-Hb or total- $\mathrm{Hb}$ [11]. First, the 5 repetitions of oxy-Hb measurement in each task were grand-averaged in each channel. In addition, we obtained the data from the last 10 seconds of each task period because hemodynamic changes as measured by fNIRS lag behind the task stimulus by a few seconds [12]. Second, to adjust for the influence of 


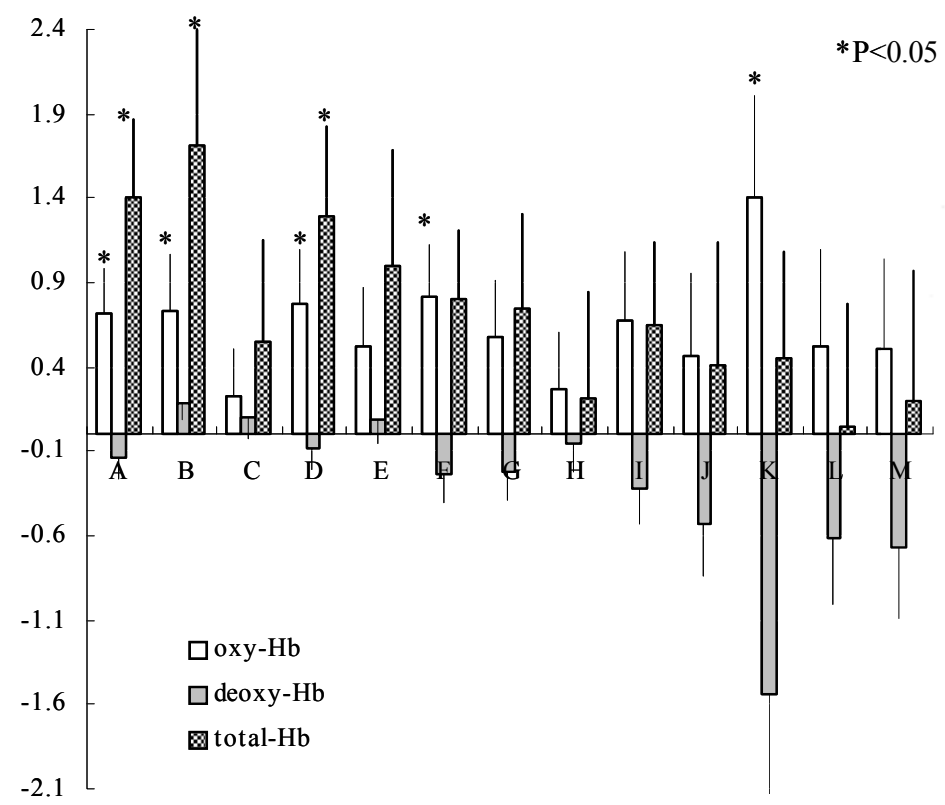

(a)

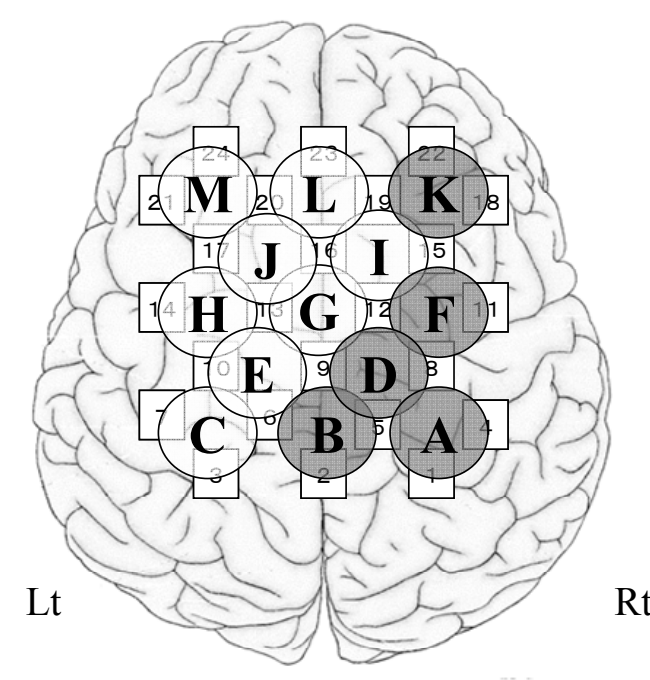

(b)

Figure 4. Mean values of effect size in regions of interest. (a) Values are mean \pm standard error. Effect size of oxy-Hb (white bars) or total- $\mathrm{Hb}$ (hatched bars) significantly increased in regions $\mathrm{A}, \mathrm{B}, \mathrm{D}, \mathrm{F}$, and $\mathrm{K}$. The effect magnitude of deoxy-Hb (black bars) tended to show negative scores. (b) The effect size of oxy-Hb located in regions of interest A, B, D, F, and K significantly increased (black circles).

differential light-path lengths among subjects or channels and to emphasize the changes of oxy-Hb level during perception of reachability, the effect size was calculated by the following formula: Effect size $=$ (mean oxy- $\mathrm{Hb}$ during perceived reachability task - mean oxy-Hb during control task) / standard deviation of oxy-Hb during the control task $[8,13]$. Finally, we established 13 regions of interest, which consisted of the 4 nearest neighboring channels (Figure 2b). The data for each region included a total of 24 effect-size values $(6$ subjects $\times 4$ channels). On the basis of these values, we used 1-sample $t$ tests to assess the regions in which the oxy-Hb level was significantly increased during the perception of reachability. Statistical significance was defined as $P<0.05$.

To examine changes of oxy- $\mathrm{Hb}$, deoxy- $\mathrm{Hb}$ and total-Hb during control task, we calculated the $\mathrm{Z}$ scores in each subject and each channel by following fomula: $Z$ score $=($ mean value during control task - mean value during pretask period of control task) / standard deviation during pretask period of control task [12]. All mean$\mathrm{Z}$ scores of oxy-Hb, deoxy-Hb and total-Hb in each channel ranged from -1 to 2 and therefore, we judged that hemoglobin levels were not changed by the control task.

\section{RESULTS}

The mean values of effect size in each channel are shown in Table 1. The effect tended to increase in the right frontal region during the perception of reachability. Statistical analysis also showed that the mean oxy- $\mathrm{Hb}$ effect size significantly increased in regions of interest A, $\mathrm{B}, \mathrm{D}, \mathrm{F}$, and $\mathrm{K}(P<0.05)$ (Figure 4).

\section{DISCUSSION}

The significant change in oxy-Hb during the perception of reachability indicated increased neural activity in the right frontal lobe. Previous researches have reported that the perception of reachability was estimated by internal mental simulation or motor imagery of the reaching movement [14-16]. Literally, motor imagery for the right hand recruits multiple cortical areas, including the left supplementary motor area, the premotor cortex [17], and the primary motor cortex [18]. As previous studies have indicated that motor imagery is related to estimation of the right arm-reach range, neural activity would be predominantly in the left frontal lobe, not in the right frontal lobe. However, since we observed activations in the right frontal lobe, our results do not support the existence of a close connection between perceived reachability and motor imagery. The lateral area of $\mathrm{Cz}$ position in the international 10-20 system corresponds with sensorimotor cortex. Significant increases of oxy-Hb level in regions of interest $\mathrm{A}, \mathrm{B}$, and $\mathrm{D}$ may be induced by the act of pushing the analog switch in the left hand during the perceived reachability task. Thus, we hypothesize that regions $\mathrm{F}$ and $\mathrm{K}$ in the right frontal lobe, located in the prefrontal cortex (PFC), are key regions in the percep- 
Table 1. The mean effect size of oxy-Hb, deoxy-Hb and total-Hb in each Channel.

\begin{tabular}{|c|c|c|c|c|c|c|}
\hline & \multicolumn{2}{|c|}{ Effect size of oxy-Hb } & \multicolumn{2}{|c|}{ Effect size of deoxy-Hb } & \multicolumn{2}{|c|}{ Effect size of total-Hb } \\
\hline $\mathrm{Ch} 1$ & 1.11 & \pm 1.68 & -0.34 & \pm 0.69 & 0.78 & \pm 0.78 \\
\hline $\mathrm{Ch} 2$ & 0.45 & \pm 1.30 & 0.12 & \pm 0.22 & 0.52 & \pm 0.50 \\
\hline Ch 3 & 0.42 & \pm 1.73 & 0.06 & \pm 0.81 & 0.75 & \pm 0.68 \\
\hline $\mathrm{Ch} 4$ & 0.43 & \pm 1.21 & -0.16 & \pm 0.56 & 0.51 & \pm 0.51 \\
\hline $\mathrm{Ch} 5$ & 0.79 & \pm 1.37 & 0.12 & \pm 0.52 & 0.70 & \pm 0.54 \\
\hline Ch 6 & 0.80 & \pm 1.80 & 0.23 & \pm 0.62 & 0.86 & \pm 0.68 \\
\hline $\mathrm{Ch} 7$ & -0.40 & \pm 0.72 & 0.19 & \pm 0.60 & 0.28 & \pm 0.42 \\
\hline Ch 8 & 0.53 & \pm 1.05 & -0.20 & \pm 0.58 & 0.49 & \pm 0.47 \\
\hline $\mathrm{Ch} 9$ & 0.87 & \pm 2.50 & 0.26 & \pm 0.63 & 1.07 & \pm 0.94 \\
\hline Ch 10 & 0.07 & \pm 1.01 & -0.09 & \pm 0.52 & 0.38 & \pm 0.43 \\
\hline Ch 11 & 0.62 & \pm 1.40 & 0.06 & \pm 0.85 & 0.73 & \pm 0.57 \\
\hline $\mathrm{Ch} 12$ & 0.90 & \pm 1.40 & -0.52 & \pm 0.64 & 0.60 & \pm 0.70 \\
\hline Ch 13 & 0.34 & \pm 1.71 & -0.07 & \pm 0.84 & 0.70 & \pm 0.69 \\
\hline $\mathrm{Ch} 14$ & -0.08 & \pm 1.56 & 0.40 & \pm 0.89 & 0.69 & \pm 0.65 \\
\hline Ch 15 & 1.22 & \pm 2.31 & -0.27 & \pm 1.19 & 1.11 & \pm 1.00 \\
\hline Ch 16 & 0.17 & \pm 1.20 & -0.55 & \pm 1.15 & 0.49 & \pm 0.70 \\
\hline Ch 17 & 0.74 & \pm 2.34 & -0.45 & \pm 0.98 & 0.90 & \pm 1.00 \\
\hline Ch 18 & 2.55 & \pm 3.43 & -3.22 & \pm 4.84 & 1.90 & \pm 2.91 \\
\hline Ch 19 & 0.39 & \pm 3.05 & 0.08 & \pm 1.25 & 1.19 & \pm 1.20 \\
\hline Ch 20 & 0.62 & \pm 3.95 & -1.06 & \pm 2.64 & 1.54 & \pm 1.88 \\
\hline $\mathrm{Ch} 21$ & 0.25 & \pm 1.89 & -0.31 & \pm 1.07 & 0.73 & \pm 0.83 \\
\hline Ch 22 & 1.47 & \pm 3.11 & -2.76 & \pm 1.89 & 0.93 & \pm 2.04 \\
\hline Ch 23 & 0.89 & \pm 3.03 & -0.92 & \pm 2.56 & 1.39 & \pm 1.56 \\
\hline $\mathrm{Ch} 24$ & 0.41 & \pm 2.61 & -0.89 & $\pm \quad 3.00$ & 1.28 & $\pm \quad 1.58$ \\
\hline
\end{tabular}

Values are mean \pm standerd diviation.

tion of reachability in a standing position; in other words, perception of the subject's own postural limits.

We also investigated the functional relationship between perceived reachability and cognitive function. Previous studies have shown that healthy young subjects tend to overestimate their own reachability while in a sitting position, although they underestimate their own reachability in a standing position. Robinovich [2] called this underestimation a potential safety factor in reducing the risk of loss of balance. This safety factor means that the process of perceiving one's own postural limits are controlled by an inhibitory neural mechanism of a motor program executing the reaching motion without risk of a fall. Shallice proposed the notion of a supervisory attentional system in the frontal lobe that selects appropriate schema among activated schemas in response to various situations, subsequently inhibiting inappropriate ones [19]. In addition, the PFC in the right hemisphere is activated by a GO/NO-GO task [20]. Therefore, the right PFC activity observed in our study may represent an inhibitory mechanism for estimating safe reaching in a standing position. Recently, Ambrose et al. reported that elderly fallers with impaired working memory overestimated their postural limits compared with those with preserved working memory [21]. They also reported that an impaired executive function might promote fall risks due to misjudgment of motor planning in daily activity. Working memory and executive function are believed to be conducted mainly in the PFC [22]. Consequently, our results indicate that working memory may be associated with perception of one's own postural limits.

\section{CONCLUSIONS}

Overestimation of postural limits is a possible risk factor for falls. Our study using fNIRS suggests that overestimation of the postural limits may result from dysfunction of the right PFC, which subsequently fails to inhibit an inappropriate motor program. Thus, activation of the right PFC may be a key factor for preventing accidental falls in the elderly and in patients with neurological disorders. Interventions to activate the right PFC could be useful for preventing accidental falls.

\section{REFERENCES}

[1] C. Carello, A. Grosofsky, F. D. Reichel, H. Y. Solomon, M. T. Turvey, (1989) Visually perceiving what is reachable. Ecological Psychology, 1, 27-54.

[2] S. N. Robinovitch, (1998) Perception of postural limits during reaching. J Mot Behav, 30(4), 352-358.

[3] S. N. Robinovitch, T. Cronin, (1999) Perception of postural limits in elderly nursing home and day care participants. J Gerontol A Biol Sci Med Sci, 54(3), B124-130; discussion B31.

[4] C. Gabbard, A. Cordova, S. Lee, (2007) Examining the 
effects of postural constraints on estimating reach. J Mot Behav, 39(4), 242-246.

[5] N. Kamata, Y. Matsuo, T. Yoneda, H. Shinohara, S. Inoue, K. Abe, (2007) Overestimation of stability limits leads to a high frequency of falls in patients with Parkinson's disease. Clin Rehabil, 21(4), 357-361.

[6] Y. Okada, K. Takatori, K. Ikuno, K. Tsuruta, K. Tokuhisa, K. Nagino, et al., (2008) Perception of postural limits and falls in community-dwelling elderly people. International Journal on Disability and Human Development, 7(1), 73-80.

[7] I. Miyai, H. C. Tanabe, I. Sase, H. Eda, I. Oda, I. Konishi, et al., (2001) Cortical mapping of gait in humans: A near-in- frared spectroscopic topography study. Neuroimage, 14(5), 1186-1192.

[8] M. Suzuki, I. Miyai, T. Ono, K. Kubota, (2008) Activities in the frontal cortex and gait performance are modulated by preparation: An fNIRS study. Neuroimage, 39(2), 600-607.

[9] M. Mihara, I. Miyai, M. Hatakenaka, K. Kubota, S. Sakoda, (2008) Role of the prefrontal cortex in human balance control. Neuroimage, 43(2), 329-336.

[10] P. W. Duncan, S. Studenski, J. Chandler, B. Prescott, (1992) Functional reach: Predictive validity in a sample of elderly male veterans. J Gerontol, 47(3), M93-98.

[11] Y. Hoshi, N. Kobayashi, M. Tamura, (2001) Interpretation of near-infrared spectroscopy signals: A study with a newly developed perfused rat brain model. J Appl Physiol 90(5), 1657-1662.

[12] Y. Otsuka, E. Nakato, S. Kanazawa, M. K. Yamaguchi, S. Watanabe, R. Kakigi, (2007) Neural activation to upright and inverted faces in infants measured by near-infrared spectroscopy. Neuroimage, 34(1), 399-406.

[13] M. L. Schroeter, S. Zysset, F. Kruggel, D. Y. von Cramon, (2003) Age dependency of the hemodynamic response as measured by functional near-infrared spectroscopy. Neuroimage, 19(3), 555-564.

[14] M. H. Fischer, (2003) Can we correctly perceive the reaching range of others? Br J Psychol, 94(Pt 4), 487-500.

[15] M. H. Fischer, (2005) Perceived reachability: The roles of handedness and hemifield. Exp Brain Res, 160(3), 283289.

[16] C. Lamm, M. H. Fischer, J. Decety, C. Lamm, (2007) Predicting the actions of others taps into one's own somatosensory representations - a functional MRI study. Neuropsychologia, 45(11), 2480-2491.

[17] P. E. Roland, B. Larsen, N. A. Lassen, E. Skinhoj, (1980) Supplementary motor area and other cortical areas in organization of voluntary movements in man. J Neurophysiol, 43(1), 118-136.

[18] C. A. Porro, M. P. Francescato, V. Cettolo, M. E. Diamond, P. Baraldi, C. Zuiani, et al., (1996) Primary motor and sensory cortex activation during motor performance and motor imagery: A functional magnetic resonance imaging study. J Neurosci, 16(23), 7688-7698.

[19] T. Shallice, (1982) Specific impairments of planning. Philos Trans R Soc Lond B Biol Sci, 298(1089), 199-209.

[20] R. Kawashima, K. Satoh, H. Itoh, S. Ono, S. Furumoto, R. Gotoh, et al., (1996) Functional anatomy of GO/NO-GO discrimination and response selection - a PET study in man. Brain Res, 728(1), 79-89.

[21] T. Liu-Ambrose, Y. Ahamed, P. Graf, F. Feldman, S. N. Robinovitch, (2008) Older fallers with poor working memory overestimate their postural limits. Arch Phys Med Rehabil, 89(7), 1335-1340.

[22] M. D’Esposito, J. A. Detre, D. C. Alsop, R. K. Shin, S. Atlas, M. Grossman, (1995) The neural basis of the central executive system of working memory. Nature, $\mathbf{3 7 8 ( 6 5 5 4 )}$, 279-81. 